\title{
Complications of Sural Nerve Biopsy in Diabetic versus Non-Diabetic Patients
}

\author{
James R. Perry and Vera Bril
}

\begin{abstract}
We compare complications from 66 sural nerve biopsies in 41 patients with diabetic peripheral neuropathy to 40 patients with neuropathy from other causes, using a retrospective telephone survey. Diabetic patients were followed for a mean of 6.8 years and non-diabetics for 5.6 years. Mild long-term pain was described by $18.9 \%$ of patients overall with no difference between groups. Mild persistent sensory symptoms, insufficient to interfere with daily activity or warrant medical therapy, were reported by $63.6 \%$ of diabetic and $27.5 \%$ of non-diabetic patients $(p<0.006)$. Wound infection and severe pain were uncommon in both groups and no different in diabetics. Significant complications of sural nerve biopsy occurred no more frequently in diabetic than in non-diabetic patients. While sural nerve biopsy plays no role in the routine evaluation of diabetic peripheral neuropathy, it may be performed without increased risk when indicated in these patients to exclude other causes of neuropathy and in the context of research trials.
\end{abstract}

\begin{abstract}
Résumé: Complications de la biopsie du nerf saphène externe chez les patients diabétiques et non diabétiques. Nous comparons, au moyen d'une enquête téléphonique rétrospective, les complications survenues lors de biopsies du nerf saphène externe chez 41 patients porteurs d'une polyneuropathie diabétique à celles survenues chez 40 patients porteurs d'une neuropathie dont l'étiologie était autre. Les patients diabétiques ont été suivis en moyenne 6.8 ans et les non diabétiques 5.6 ans. A long terme, $18.9 \%$ de tous les patients accusaient une légère douleur, sans différence entre les groupes. $63.6 \%$ des diabétiques et $27.5 \%$ des non diabétiques $(\mathrm{p}<0.006)$ ont rapporté des symptômes sensitifs légers, persistants, qui n'entravaient pas les activitiés quotidiennes et ne justifiaient pas un traitement médical. Une infection de la plaie et des douleurs sévères étaient rares dans les deux groupes, sans différence chez les diabétiques. Les complications majeures de la biopsie du nerf saphène externe ne sont pas survenues plus fréquemment chez un groupe que chez l'autre. Bien que la biopsie du nerf saphène externe ne soit pas un examen de routine dans l'évaluation de la neuropathie diabétique, elle peut être effectuée sans risque accru quand il y a indication chez ces patients pour exclure d'autres causes de neuropathie et dans le contexte d'études cliniques.
\end{abstract}

Can. J. Neurol. Sci. 1994; $21: 34-37$

Sural nerve biopsy (SNBx) is widely used as an adjunct in the investigation of peripheral neuropathy (PN). More recently, SNBx has been used in research protocols, particularly in diabetic PN, as a means of end-point analysis and to allow morphologic study.' Decisions to biopsy in an individual patient must be based upon judgment of the potential yield versus complications of the procedure. While the sequelae of SNBx have not been well documented, many authors believe that persistent symptoms are unusual ${ }^{2,3}$ despite small retrospective series that have shown long-term disturbances in a high proportion of patients. ${ }^{4 \cdot 6}$

Patients with diabetes mellitus (DM) are subject to surgical complications including poor wound healing and infection. DM is prevalent in neurology clinics, and SNBx may be considered in these patients when other causes of PN are suspected. The outcome of SNBx has never been evaluated in this population. For this reason, we compared the sequelae of SNBx in patients with diabetic PN to patients with neuropathy from other causes.

\section{Methods \\ Patient Selection}

The diabetic group consisted of 41 patients with diabetic PN enrolled in clinical trials of aldose-reductase inhibitors. SNBx was performed at study entry in all patients and 25 had repeat biopsy at the study end for a total of 66 biopsies in 41 patients. All had insulin-dependent or non-insulin-dependent DM according to WHO criteria, clinical PN and absence of other causes of neuropathy. Clinical details of many of these patients have been previously described. ${ }^{7}$ PN was defined by symptoms and signs

From the Neuromuscular Laboratory, Toronto General Division. The Toronto Hospital, Toronto.

RECEIVED FEBRUARY 2, 1993. ACCEPTED IN FINAL FORM OCTOBER 13, 1993.

This work was presented in abstract form at the Scientific Program, American Academy of Neurology Annual Meeting, New York, New York, April 25-May I. 1993.

Reprint requests to: Vera Bril, M.D., Division of Neurology, Eaton North 11-209. The Toronto Hospital, General Division, 200 Elizabeth Street. Toronto, Ontario, Canada M5G 2C4 
consistent with distal symmetric polyneuropathy and confirmed by the presence of elevated tactile or thermal thresholds and abnormal nerve conduction studies.

The non-diabetic group consisted of 40 patients who underwent SNBx as part of diagnostic work-up for PN due to causes other than DM. Patients included were those referred to and examined at the Toronto General neuromuscular laboratory. Between 1982 and 199l, 64 patients had SNBx. Since then, 8 have died, 6 have developed DM, and 10 were lost to follow-up leaving 40 patients who are reported here. All had normal blood glucose and hemoglobin A1C levels at both initial presentation and over the course of follow-up. Biopsy diagnoses in these 40 patients were: non-specific axonal neuropathy (23), demyelinating PN (6), vasculitis (6), and one each of normal, tomaculous, leprosy, amyloid, and lymphomatoid granulomatosis.

\section{Biopsy Protocol}

Standard techniques were used for the biopsies. ${ }^{8.9}$ Thirty-six of the biopsies in diabetic patients were fascicular as described elsewhere ${ }^{7}$ and thirty were whole nerve biopsies. All of these biopsies were performed by the same neurosurgeon at a similar anatomical site in each patient. All of the non-diabetic group had whole nerve biopsies performed by a consultant neurosurgeon at our institution. Twenty-five patients in the diabetic group had a repeat biopsy following the drug trial and had either a repeat ipsilateral biopsy ( 22 patients) by extending the incision proximally, or a contralateral biopsy (3 patients). Patients were instructed to keep their leg elevated, wear a pressure dressing, and minimize weight bearing for 48 hours following surgery. Patients were assessed during the post-operative period to assess wound healing.

\section{Symptom Survey}

All patients were assessed with a standardized questionnaire for the presence of post-operative symptoms including ongoing pain, sensory disturbance, and wound infection. The diabetic patients were seen at regular intervals as part of ongoing clinical trials and the non-diabetic group was assessed by a single examiner. For the purposes of this study a telephone survey was conducted to ascertain the presence of symptoms following biopsy. The survey was conducted at a mean of 6.8 and 5.6 years following biopsy for diabetic and non-diabetic patients respectively. All patients contacted responded to the survey.

Symptoms of pain and sensory phenomena were recorded. Descriptors such as burning, jabbing, aching, and allodynia were recorded as pain while spontaneous or tactile-induced parathesiae, complaints of new numbness or altered sensation were included as sensory symptoms. Immediate pain included symptoms occurring up to 4 weeks post-operatively. Persistent symptoms were those present (intermittently or consistently) at the time of the survey. Symptoms were recorded as mild if they were described as a nuisance only and did not interfere in any way with the patient's daily living. If the symptoms disturbed sleep, limited activities, or required the use of medication (including simple analgesics), they were recorded as severe. Only those symptoms that were ipsilateral to the biopsy site and felt not to be related to progression of the underlying neuropathy were included. Wound infections were generally documented in follow-up or by the survey. These were classified as mild if they responded to local measures or oral antibiotics, or severe if IV antibiotics or surgical treatment were needed.
Results were analyzed using standard statistical measures including ANOVA and Chi square.

\section{RESULTS}

Characteristics of the patient population are shown in Table 1. Sex, age at time of biopsy, and length of follow-up after biopsy were no different between groups. Diabetic patients had a longer mean duration of symptoms prior to SNBx than non-diabetics. This difference is likely due to entry criteria for some of the drug trials requiring a symptom duration of at least 6 months, whereas several non-DM patients had acute neuropathies (for example due to vasculitis).

Results of the post-biopsy symptom survey are shown in Table 2. Significant differences between the two groups were seen only for the presence of persistent mild sensory symptoms. Immediate pain was severe in only $2.7 \%$ of the entire cohort and persistent in none. Severe sensory symptoms, commonly described as burning and tingling exacerbated by both movement and touch, were reported by two non-diabetic patients. One of these patients had resumed weight-bearing immediately after biopsy causing wound dehiscence. Severe infection occurred only in one non-diabetic patient who developed a chronic draining stitch abscess requiring revision.

Table 1. Patient Characteristics.

\begin{tabular}{lcc}
\hline & $\begin{array}{c}\text { Diabetic group } \\
(\mathbf{n}=\mathbf{4 1})\end{array}$ & $\begin{array}{c}\text { Non-diabetic group } \\
(\mathbf{n}=\mathbf{4 0})\end{array}$ \\
\hline $\begin{array}{l}\text { Sex } \\
\begin{array}{l}\text { Mean age } \\
\text { (years) }\end{array}\end{array}$ & $29 \mathrm{M}, 12 \mathrm{~F}$ & $27 \mathrm{M}, 13 \mathrm{~F}$ \\
$\begin{array}{l}\text { Mean duration } \\
\text { of neuropathy } \\
\text { (years) }\end{array}$ & $49.3(+/-12.6)$ & $48.7(+/-8.4)$ \\
$\begin{array}{l}\text { Mean follow-up } \\
\text { after biopsy } \\
\text { (years) }\end{array}$ & $2.2(+/-2.1)$ & $0.5(0-5.0)$ \\
\hline
\end{tabular}

Table 2. Results of the Post-Biopsy Symptom Survey.

\begin{tabular}{llccc}
\hline & & All patients & Diabetic & Non-Diabetic \\
\hline $\begin{array}{l}\text { total \# of } \\
\text { biopsies }\end{array}$ & & 106 & 66 & 40 \\
immediate & none & $73(68.9 \%)$ & $42(63.6 \%)$ & $31(77.5 \%)$ \\
pain & mild & $31(29.2 \%)$ & $23(34.8 \%)$ & $8(20.0 \%)$ \\
& severe & $2(1.9 \%)$ & $1(1.5 \%)$ & $1(2.5 \%)$ \\
persistent & none & $86(81.1 \%)$ & $55(83.3 \%)$ & $31(77.5 \%)$ \\
pain & mild & $20(18.9 \%)$ & $11(16.7 \%)$ & $9(22.5 \%)$ \\
& severe & 0 & 0 & 0 \\
persistent & none & $51(48.1 \%)$ & $24(36.4 \%)^{*}$ & $27(67.5 \%)$ \\
sensory & mild & $53(50.0 \%)$ & $42(63.6 \%)^{*}$ & $11(27.5 \%)$ \\
symptoms & severe & $2(1.9 \%)$ & 0 & $2(5.0 \%)$ \\
wound & none & $101(95.3 \%)$ & $64(97.0 \%)$ & $37(92.5 \%)$ \\
infection & mild & $4(3.8 \%)$ & $2(3.0 \%)$ & $2(5.0 \%)$ \\
& severe & $1(0.9 \%)$ & 0 & $1(2.5 \%)$ \\
\hline
\end{tabular}

$*=p<0.006$ 
Mild symptoms were commonly reported by both groups. Overall, $18.9 \%$ of patients reported persistent mild pain with descriptors such as jabbing and aching with a trend to more complaints in the non-diabetic group. No patient in either group reported new burning following biopsy and none required re-exploration for suspected neuroma formation. Mild persistent sensory disturbances were noted by $50 \%$ of patients overall and described as a lack of sensation with tactile-induced dysesthesia or uncomfortable feelings in the lateral foot. Patients with DM reported these types of symptoms more frequently $(63.6 \% \mathrm{vs}$ $27.5 \%, p<0.006$ ); however, of these symptomatic diabetic patients, 22 had repeat ipsilateral biopsies and were therefore surveyed following the effects of both procedures. Because the patients were surveyed many years after the procedures and the majority had minimal symptoms to report, it was not possible to discern the effect from each biopsy on an individual basis.

To determine the importance of pre-existing sensory neuropathy to the development of later sensory complications, the non-diabetic group was analyzed separately. Diabetic patients were not included since they all had prior sensory symptoms. Of the group of 40 non-diabetic patients, 32 had sensory symptoms as part of their neuropathy, with 13 developing new sensory complaints following biopsy. In contrast, none of the 8 patients without pre-biopsy symptoms developed persistent sensory sequelae $(\mathrm{p}<0.05)$.

Overall, $40.6 \%$ of patients would refuse to undergo repeat SNBx, with no difference between groups (data not shown). Reasons for refusal were lack of symptomatic improvement or a definitive diagnosis in 10 patients while others cited inconvenience, discomfort, presence of the surgical scar, and wound infection in decreasing order of frequency.

\section{Discussion}

This is the first report, to our knowledge, to evaluate complications of SNBx specifically in a population of diabetic patients. Depending upon the study protocol, the diabetic patients had either whole or fascicular SNBx. Fascicular biopsies are widely felt to be inadequate for routine pathologic examination since processes such as vasculitis or amyloidosis may be focal and missed with limited examination. ${ }^{10.11}$ Differences in outcome between these two techniques have not been well defined. Persistent sensory loss and sensory disturbances are felt by Dyck $^{12}$ and Thomas ${ }^{3}$ to be less common following fascicular SNBx; however, this experience is largely anecdotal. Pollock et al. found no difference in long-term sensory complaints or sensory loss when comparing these techniques directly in small numbers of patients. ${ }^{10}$ Overall, in that study, 11 of 16 patients reported "unpleasant" sensory symptoms following biopsy. Since all of our non-diabetic patients had whole SNBx we were unable to compare our results according to the type of procedure.

In studies of PN due to causes other than diabetes, Poburski ${ }^{4}$ evaluated 24 of 82 available patients and found "considerable" discomfort in $21 \%$ following SNBx, but speculated that this incidence would be lower without selection bias. Neundorfer described 56 patients after whole nerve biopsy ${ }^{6}$ with a $25 \%$ incidence of persistent pain and a 30\% incidence of sensory symptoms. Pre-operative sensory abnormalities were present in 53\% of these patients; however, no attempt was made to correlate these with outcome. In our group of non-diabetic patients, prior sensory symptoms strongly predicted the development of postoperative complaints. Overall, $19 \%$ of our patients reported persistent pain and $50 \%$ described persistent sensory disturbances suggesting a higher complication rate than previously reported. ${ }^{4.6 .12}$ One reason for this is inclusion of many patients with relatively minor symptoms which, by their own admission, would not have been reported to a physician outside of this study. Less than $2 \%$ of patients overall stated that their symptoms were severe enough to interfere with their activities or require the use of medications for control. Assessment of individual risk factors for these complications was not possible given the retrospective nature of our data.

Long-term sequelae were no different between groups with the exception of mild sensory complaints in diabetics. Several confounding factors affect interpretation of this result. Many of the diabetic patients had a repeat ipsilateral biopsy and therefore would be expected to have a greater degree of sensory loss with interruption of ongoing nerve regeneration plus the unknown risk of a second procedure. Although symptoms were included only if clearly worse on the side of the surgery and in the region of the scar or in the distribution of the sural nerve, it may be difficult for both physician and patient to avoid confusing these symptoms with those of progressive neuropathy. All patients in the diabetic group had advanced neuropathy and this may have biased reporting of further symptoms. It is also possible that inclusion in closely-monitored clinical trials may have led to over-reporting of symptoms. It should be emphasized that these mild symptoms were, in general, of no consequence to the patient. Infection rates were very low overall and similar to those in previous reports. ${ }^{8.11 .13}$ Although wound healing and infection are a concern in patients with DM, we found no evidence for an increased rate of such complication.

Approximately $40 \%$ of patients stated that they would not undergo SNBx again. This is higher than the 10 of 97 patients reported by Dyck. ${ }^{2}$ In our patients, the most common reasons for reluctance were a lack of symptomatic improvement and a biopsy result that did not lead to a diagnosis or influence management. Persistent mild sensory symptoms were not a factor reported by the patients in this regard. While diabetics with severe PN may be at slightly increased risk of minor sensory sequelae following SNBx there is no greater risk of significant sensory complications, pain, or wound infection when compared to patients with neuropathy from other causes. SNBx plays no role in the routine evaluation of diabetic PN; however, it can be performed without increased risk of painful sequelae or infection if other causes of neuropathy are suspected or in the context of research studies.

\section{REFERENCES}

1. Proceedings of a consensus development conference on standardized measures in diabetic neuropathy. Neurology 1992; 42: 1823-1939.

2. Dyck PJ, Kames J, Lais A. Pathologic alterations of the peripheral nervous system of humans. In: Dyck PJ, Thomas PK, Lambert EH, Bunge R, eds. Peripheral Neuropathy, second ed. Philadelphia: Sanders, 1985: 771-777.

3. Thomas PK. The quantitation of nerve biopsy findings. J Neurol Sci 1970; 11:285-295.

4. Poburski R, Malin J-P, Stark E. Sequelae of sural nerve biopsies. Clin Neurol Neurosurg 1985; 87: 193-197. 
5. Solders G. Discomfort after fascicular sural nerve biopsy. Acta Neurol Scand 1988; 77: 503-504.

6. Neundorfer B, Grahamm F, Engelhardt A, Harte U. Postoperative effects and value of sural nerve biopsies: a retrospective study. Eur Neurol 1990; 30: 350-352.

7. Sima AAF, Bril V, Nathaniel V, et al. Regeneration and repair of myelinated fibres in sural-nerve biopsy specimens from patients with diabetic neuropathy treated with sorbinil. N Engl J Med 1988; 319: 548-555.

8. Asbury AK, Connolly ES. Sural nerve biopsy. Technical note. J Neurosurg 1973; 38: 391-392.

9. Dyck PJ, Lofgren EP. Method of fascicular biopsy of human peripheral nerve for electrophysiologic and histologic study. Mayo Clin Proc 1966; 41: 778-784.
10. Pollock M, Nukada H, Taylor P, Donaldson I, Carroll G. Comparison between fascicular and whole sural nerve biopsy. Ann Neurol 1983; 13: 65-68.

11. Oh SJ. Diagnostic usefulness and limitations of the sural nerve biopsy. Yonsei Med J 1990; 31: 1-26.

12. Dyck PJ, Lofgren EP. Nerve biopsy: choice of nerve, methods, symptoms, and usefulness. Med Clin North Am 1968; 52: 885893.

13. Appenzeller O, Snyder RD, Kornfeld M. Sural nerve biopsies in pediatric neurological disorders. Dev Med Child Neurol 1970; 12: $42-48$. 